\section{$\Omega$}

Renata L. Riha

(1)

Sleep Medicine, Royal Infirmary Edinburgh, Edinburgh, UK.

\title{
Breathe: the influence of gender on respiratory conditions commencing in childhood
}

The influence of gender on the evolution of both rare and common chronic conditions, their management and response to treatment has been a poorly studied area in which we are in catching up as pulmonologists. Perhaps psychiatrists and psychologists have recognised that there are gender differences in behaviour and response to illness, which evolve over time from childhood into adulthood, but there is far less research in this area in internal medicine, let alone surgery!

In this issue, we have a number of excellent reviews tackling uncommon illnesses: bronchopulmonary dysplasia [1], cystic fibrosis (CF) and non-CF bronchiectasis [2], and an online exclusive focusing on $\alpha_{1}$-antitrypsin deficiency. In addition, we have an outstanding physiology masterclass on the physiological differences in respiratory function between the two sexes [3]. At present, there is no really good information on those who are transitioning between genders during adolescence and the influence of hormone treatments on their lungs, something that we will probably come to understand over time and with greater awareness.

For those of you who regularly prescribe and apply noninvasive ventilation (NIV) to your patients, MukherJee et al. [4] review the impact of the work that led to its use outside intensive care and high-dependency units. In many hospitals throughout the world, NIV is a life-saving, and also at times palliative, procedure that is routinely performed in a ward-level setting. This would not have been possible without clinical trials.

And apropos of clinical trials, members of a European Respiratory Society funded clinical research collaboration have developed a protocol for a prospective pan-European observational study of paediatric severe asthma and patient registry, which aims to improve recruitment to paediatric studies in respiratory medicine [5]. In addition, we have a short paper incorporating some handy hints on how to obtain informed consent from patients [6]. If we are to progress in our understanding of respiratory disease and its management, it is essential that our patients help us along the way through trial participation. In return, we are beholden to them in terms of minimising harm: primum non nocere!

Once again, I would like to thank the outstanding editorial team in Sheffield and all the contributors to this issue who have given of their time and experience. After a bleak and seemingly endless winter, spring has finally broken through for most of us in the Northern hemisphere. So here's to a long, sunny summer! Happy reading!
Cite as: Riha RL. Breathe: the influence of gender on respiratory conditions commencing in childhood. Breathe 2018; 14: 85-86. 


\section{Conflict of interest}

None declared.

\section{References}

1. Kotecha SJ, Lowe J, Kotecha S. Does the sex of the preterm baby affect respiratory outcomes? Breathe 2018; 14 100-107.

2. Vidaillac C, Yong VFL, Jaggi TK, et al. Gender differences in bronchiectasis: a real issue? Breathe 2018; 14 108-121.

3. LoMauro A, Aliverti A. Sex differences in respiratory function. Breathe 2018; 14: 131-140.
4. Mukherjee R, Nenna R, Turner A. Early ward-based acute noninvasive ventilation: a paper that changed practice. Breathe 2018; 14: 153-155.

5. Liu NM, van Aalderen W, Carlsen KCL, et al. Severe Paediatric Asthma Collaborative in Europe (SPACE): protocol for a European registry. Breathe 2018; 14: 93-98.

6. Manti S, Licari A. How to obtain informed consent for research. Breathe 2018; 14: 145-152. 